\title{
ERRATUM
}

\section{An octave-bandwidth negligible-loss radiofrequency metamaterial}

Erik Lier, Douglas H. Werner, Clinton P. Scarborough, Qi Wu and Jeremy A. Bossard

Nature Materials doi:10.1038/nmat2950 (2011); published online: 30 January 2011; corrected online: 3 February 2011.

In the version of this Article originally published online, the values on the $y$-axis of Fig. $2 \mathrm{~d}$ were incorrectly aligned. This error has now been corrected in all versions of the text. 